\title{
EXPLICIT SOLUTIONS TO PHASE CHANGE PROBLEMS*
}

\author{
BY \\ A. D. SOLOMON, D. G. WILSON(Union Carbide Corporation, Nuclear Division) \\ AND \\ V. ALEXIADES(Union Carbide Corporation, Nuclear Division and University of Tennessee)
}

\begin{abstract}
We examine two heat transfer and phase change problems having explicit solutions. The first involves melting of an initially cold material and clarifies the meaning of a recent result of Tarzia [5]. The second concerns a model of binary alloy solidification which, in some cases, is seen to be incorrect.
\end{abstract}

Introduction. This paper was motivated by a recent discussion of Tarzia [5]. In it we consider the heating of a semi-infinite slab of material whose temperature is initially below the melting point, by a heat flux of the form $h_{0} / \sqrt{t}$ at its surface. The fact that if $h_{0}$ is too small, then no melting will occur [e.g., the Neumann solution does not exist] is derived in a complicated fashion; the actual reason for this result is that if $h_{0}$ is too small, the slab temperature is never raised up to the melting point, and hence melting is never initiated. This is the subject of Sec. 1.

The fact that problems that appear to be reasonable may not have a solution is clear in such cases as that of Sec. 1. A similar situation has been noted earlier in [6] for supercooling. However, problems of greater subtlety exist and are little recognized as possibly lacking solutions. One such problem arises in the process of binary alloy solidification. In [3] such a problem is formulated and an explicit, Neumann-like solution is provided for it. Subsequent studies (e.g., [2, 4]) have been based upon this formulation. Upon close examination, one finds that this solution in fact may not solve the original problem, in the sense that it produces a "mushy zone" in place of a sharp phase change front. This is the subject of Sec. 2 .

The nomenclature used is given after Sec. 2 .

1. On the paper of Tarzia. Consider a semi-infinite slab $x \geqslant 0$ of material that melts at temperature $T_{c r}$. Suppose that it is initially cold at the uniform temperature $T_{s}<T_{c r}$. If a

* Received May 20, 1982.

Research sponsored by the Applied Mathematical Sciences Research Program, Office of Energy Research, U.S. Department of Energy under contract W-7405-eng-26 with the Union Carbide Corporation. 
constant temperature $T_{L}>T_{c r}$ is imposed at $x=0$, then instantaneous melting occurs with a melt front $x=X(t)$ emanating from $x=0: X(0)=0$. However, what happens if a heat flux $q_{0}$ is imposed at $x=0$ ? If $q_{0}$ is constant, then melting is not immediately begun at $t=0$, for the material temperature $T(0, t)$ must be raised from $T_{s}$ to $T_{c r}$ before melting begins. Recalling $[1$, p. 75$]$ that

$$
T(0, t)=T_{s}+\frac{2 q_{0}}{K_{s}} \sqrt{\frac{\alpha_{s} t}{\pi}}
$$

we find that $T(0, t)$ attains the value $T_{c r}$ at time

$$
t_{0}=\frac{\pi}{\alpha_{s}}\left[\frac{\left(T_{c r}-T_{s}\right) K_{s}}{2 q_{0}}\right]^{2} .
$$

What happens if $q_{0}$ is a function of $t$ ? In the special case where $q_{0}=h_{0} t^{n / 2}$ for $n=-1,0,1, \ldots$, we find

$$
T(0, t)=\frac{h_{0} \sqrt{\alpha_{s}} \Gamma\left(\frac{1}{2} n+1\right)}{K_{s} \Gamma\left(\frac{1}{2} n+\frac{3}{2}\right)} t^{(n+1) / 2}+T_{s} ;
$$

if $n=0,1,2, \ldots$, then $T(0, t)$ will only reach $T_{c r}$ at the time $t_{0}$ for which $T\left(0, t_{0}\right)=T_{c r}$ or

$$
t_{0}=\left[\frac{K_{s} \Gamma\left(\frac{1}{2} n+\frac{3}{2}\right)}{h_{0} \sqrt{\alpha_{s}} \Gamma\left(\frac{1}{2} n+1\right)}\left(T_{c r}-T_{s}\right)\right]^{1 /((n+1) / 2)},
$$

whence $X\left(t_{0}\right)=0$. However, suppose that $n=-1$. Now

$$
T(0, t)=\frac{h_{0} \sqrt{\alpha_{s}} \Gamma\left(\frac{1}{2}\right)}{K_{s}}+T_{s}=\frac{h_{0} \sqrt{\pi \alpha_{s}}}{K_{s}}+T_{s}
$$

and so $T(0, t)$ is constant in time. However, if this constant temperature is less than $T_{c r}$, the solid can never reach its melt temperature. Thus melting will only occur if

$$
\frac{h_{0} \sqrt{\pi \alpha_{s}}}{K_{s}}+T_{s}>T_{c r}
$$

or

$$
h_{0}>\frac{K_{s}}{\sqrt{\pi \alpha_{s}}}\left(T_{c r}-T_{s}\right)
$$

which is the condition derived in [5].

2. On a model of binary alloy solidification. Consider a semi-infinite slab of a binary alloy consisting of two components $A, B$. Let $C$ be the concentration of " $A$ ". Suppose that solidification of the alloy is governed by an equilibrium phase diagram consisting of a liquidus curve $T=f_{L}(C)$, and a solidus curve $T=f_{S}(C), 0<C<1$. As in [3] we assume $f_{L}, f_{S}$ to be monotonically increasing, $f_{L}(C)>f_{S}(C)$ and $f_{L}(0)=f_{S}(0)=T_{c r}^{A}, f_{L}(1)=f_{S}(1)$ $=T_{c r}^{B}$. Material is in its solid state if $T \leqslant f_{S}(C)$ and liquid if $T \geqslant f_{L}(C)$. If $f_{S}(C)<T<$ 
$f_{L}(C)$, then the material state is not well defined; it is then referred to in such terms as "mushy", "heterogeneous" and "unstable".

Consider the following process. The semi-infinite alloy is initially liquid at constant temperature $T_{\text {init }}$ and concentration $C_{\text {init }}$, for which $T_{\text {init }} \geqslant f_{L}\left(C_{\text {init }}\right)$. Beginning at time $t=0$, a cold temperature $T_{\text {wall }}<T_{c r}^{A}$ is imposed at $x=0$. Freezing occurs with, in principle, a sharp phase change front $x=X(t)$ separating solid alloy $(x<X(t))$ from liquid alloy $(x>X(t))$.

A mathematical formulation of the solidification process is given in [3] as follows:

Problem: Find temperature $T(x, t)$, concentration $C(x, t)$ and phase change front $x=X(t)$, for which:

$$
\begin{gathered}
C_{t}=D_{S} C_{x x}, \quad 0<x<X(t), \\
C_{t}=D_{L} C_{x x}, \quad x>X(t), \\
T_{t}=\alpha_{s} T_{x x}, \quad x<X(t), \\
T_{t}=\alpha_{L} T_{x x}, \quad x>X(t), \\
T_{c r}=T(X(t), t)=f_{S}[C(X(t)-, t)]=f_{L}[C(X(t)+, t)], \\
X^{\prime}(t)[C(X(t)-, t)-C(X(t)+, t)] \\
=-D_{S} C_{x}(X(t)-, t)+D_{L} C_{x}(X(t)+, t), \\
\rho H X^{\prime}(t)=-K_{L} T_{x}(X(t)+, t)+K_{S} T_{x}(X(t)-, t), \\
T(0, t)=T_{\text {wall }}, \quad t>0, \\
T(x, 0)=T_{\text {init }}, \quad x>0, \\
C(x, 0)=C_{\text {init }}, \quad x>0, \\
C_{x}(0, t)=0, \quad t>0, \\
T_{\text {init }}>f_{L}\left(C_{\text {init }}\right), \\
T_{\text {wall }}<T_{c r}^{A}, \\
X(0)=0 .
\end{gathered}
$$

THEOREM. A solution to this problem exists for all choices of initial and boundary conditions and thermal and diffusion parameters. Moreover, for this solution the solidus and liquidus concentrations

$$
C_{S}=C(X(t)-, t), \quad C_{L}=C(X(t)+, t)
$$

as well as the phase change temperature

$$
T_{c r}=T(X(t), t)
$$

are unchanged in time.

Proof. We note first that solutions to the heat and diffusion equations (1a)-(lb) can be expressed in the form

$$
\begin{gathered}
C(x, t) \equiv C_{s}=\text { const., } \quad x<X(t), \\
T(x, t)=T_{\text {wall }}+\left(T_{c r}-T_{\text {wall }}\right) \operatorname{erf}\left(x / 2 \sqrt{\alpha_{s} t}\right) / \operatorname{erf} \lambda, \quad x<X(t),
\end{gathered}
$$




$$
\begin{array}{r}
C(x, t)=C_{\text {init }}+\left(C_{L}-C_{\text {init }}\right) \operatorname{erfc}\left(x / 2 \sqrt{D_{L} t}\right) / \operatorname{erfc}\left(\lambda \sqrt{\alpha_{S} / D_{L}}\right), \\
x>X(t), \\
T(x, t)=T_{\text {init }}+\left(T_{c r}-T_{\text {init }}\right) \operatorname{erfc}\left(x / 2 \sqrt{\alpha_{L} t}\right) / \operatorname{erfc}\left(\lambda \sqrt{\alpha_{S} / \alpha_{L}}\right), \\
x>X(t),
\end{array}
$$

where

$$
T_{c r}=f_{S}\left(C_{S}\right)=f_{L}\left(C_{L}\right)
$$

the interface is of the form

$$
X(t)=2 \lambda \sqrt{\alpha_{s} t}
$$

where $\lambda$ and $T_{c r}$ (or $C_{S}, C_{L}$ ) are yet to be determined. Substitution into the interface conditions $(\mathrm{lf}),(\mathrm{lg})$ yields the equations

$$
T_{c r}=\left(A_{1}+A_{2}\right) /\left(A_{3}+A_{4}\right)
$$

where

$$
\begin{aligned}
& A_{1}=\rho H+\left[K_{S} T_{\text {wall }} / \sqrt{\pi} \alpha_{S} \lambda e^{\lambda^{2}} \operatorname{erf} \lambda\right] \\
& A_{2}=K_{L} T_{\text {init }} /\left[\sqrt{\pi \alpha_{L} \alpha_{S}} \lambda e^{\lambda^{2} \alpha_{S} / \alpha_{L}} \operatorname{erfc}\left(\lambda \sqrt{\alpha_{S} / \alpha_{L}}\right)\right], \\
& A_{3}=K_{S} /\left[\sqrt{\pi} \alpha_{S} \lambda e^{\lambda^{2}} \operatorname{erf} \lambda\right] \\
& A_{4}=K_{L} /\left[\sqrt{\pi \alpha_{L} \alpha_{S}} \lambda e^{\lambda^{2} \alpha_{S} / \alpha_{L}} \operatorname{erfc}\left(\lambda \sqrt{\alpha_{S} / \alpha_{L}}\right)\right],
\end{aligned}
$$

and

$$
\left(C_{\text {init }}-C_{L}\right) /\left(C_{S}-C_{L}\right)=\sqrt{\pi} \lambda \sqrt{\alpha_{S} / D_{L}} e^{\lambda^{2} \alpha_{S} / D_{L}} \operatorname{erfc}\left(\lambda \sqrt{\alpha_{S} / D_{L}}\right) .
$$

Let us write (4a) as

$$
T_{c r}=W_{1}(\lambda),
$$

and let us denote the functional dependence of $T_{c r}$ on $\lambda$ via (3a), (4b) by

$$
T_{c r}=W_{2}(\lambda) \text {. }
$$

We claim that there exists a $\lambda$ such that $W_{1}(\lambda)=W_{2}(\lambda)$, which will prove the Theorem. To see this, let us study the behavior of $W_{1}(\lambda)$ and $W_{2}(\lambda)$.

A short calculation (cf. [3, pp. 55-56]) shows that $W_{1}(\lambda)$ is an increasing function of $\lambda$. As $\lambda \rightarrow 0, W_{1}(\lambda) \rightarrow T_{\text {wall }}$. On the other hand, using the relation

$$
\sqrt{\pi} z e^{z^{2}} \operatorname{erfc}(z) \rightarrow 1 \quad \text { as } z \rightarrow \infty,
$$

we obtain

$$
W_{1}(\lambda) \rightarrow \frac{\rho H+K_{L} T_{\text {init }} / \alpha_{L}}{K_{L} / \alpha_{L}}=T_{\text {init }}+\frac{H}{c_{L}} \quad \text { as } \lambda \rightarrow \infty .
$$


As $\lambda \rightarrow 0,(4 \mathrm{~b})$ implies that

$$
\frac{C_{\text {init }}-C_{L}}{C_{S}-C_{L}} \rightarrow 0
$$

or $C_{L} \rightarrow C_{\text {init }}$, whence $W_{2}(\lambda)=f_{L}\left(C_{L}\right)$ must tend to the value $T_{L}^{*}=f_{L}\left(C_{\text {init }}\right)>T_{c r}^{A}$. Similarly, as $\lambda \rightarrow \infty$,

$$
\frac{C_{\text {init }}-C_{L}}{C_{S}-C_{L}} \rightarrow 1
$$

or $C_{S} \rightarrow C_{\text {init }}$. Hence $W_{2}(\lambda)=f_{S}\left(C_{S}\right)$ must tend to the value $T_{S}^{*}=f_{S}\left(C_{\text {init }}\right)<T_{\text {init }}$.

Thus we see that the continuous functions $W_{1}(\lambda)$ and $W_{2}(\lambda)$ satisfy:

$$
W_{1}(0)=T_{\text {wall }}<T_{c r}^{A}<T_{L}^{*}=W_{2}(0)
$$

and

$$
W_{2}(\infty)=T_{S}^{*}<T_{\text {init }}<T_{\text {init }}+\frac{H}{C_{L}}=W_{1}(\infty) .
$$

Therefore there exists at least one value of $\lambda$ such that $W_{1}(\lambda)=W_{2}(\lambda)$ which provides a solution of (3), (4) and completes the proof of the Theorem.

But does this imply that our problem has a solution? We claim that the reasonable condition that $X(t)$ separate solid $(x<X(t))$ from liquid $(x>X(t))$ may not be satisfied by the solution. Let us see how this can be. Consider the behavior of our solution in the physically reasonable case in which the material diffusivity in the liquid is much smaller than the solid thermal diffusivity, $D_{L} / \alpha_{S} \rightarrow 0$.

LEMma. $\lambda$ is bounded away from zero as $\alpha_{S} / D_{L} \rightarrow \infty$.

For if not, there would be a sequence $\left\{\lambda_{n}\right\}$, with $\lambda_{n} \rightarrow 0$. But then there would be a sequence $\left\{T_{c r}^{n}\right\}$ of $T_{c r}$ values for which $T_{c r}^{n} \rightarrow T_{\text {wall }}$, which is not possible since $T_{\text {wall }}<T_{c r}^{A}$.

Thus as $\alpha_{S} / D_{L} \rightarrow \infty, \lambda \alpha_{S} / D_{L} \rightarrow \infty$, and so $C_{S}\left(T_{c r}\right) \rightarrow C_{\text {init }}$. Hence $T_{c r} \rightarrow f_{S}\left(C_{\text {init }}\right)$, and so the limiting solution for $\alpha_{S} / D_{L} \rightarrow \infty$ obeys

$$
\begin{aligned}
& C_{S} \equiv C_{\text {init }}, \\
& T_{c r} \equiv f_{S}\left(C_{\text {init }}\right),
\end{aligned}
$$

while $\lambda$ tends to the solution $\lambda^{*}$ for this choice of $T_{c r}$. Thus $X(t)$ tends to the limiting interface position $X^{*}(t)=2 \lambda^{*} \sqrt{\alpha_{s} t}$.

Now consider what happens to the liquid concentration for fixed $t>0$. Let $x \geqslant X^{*}(t)$ $+\delta$ for any fixed $\delta>0$. For $D_{L} \rightarrow 0$, again using (5), we have

$$
\sqrt{\pi} \frac{x}{2 \sqrt{D_{L} t}} e^{x^{2} / 4 D_{L} t} \operatorname{erfc}\left(x / 2 \sqrt{D_{L} t}\right) \rightarrow 1
$$

and

$$
\sqrt{\pi} \lambda \sqrt{\alpha_{S} / D_{L}} e^{\lambda^{2} \alpha_{S} / D_{L}} \operatorname{erfc}\left(\lambda \sqrt{\alpha_{S} / D_{L}}\right) \rightarrow 1
$$


whence

$$
\left\{\frac{\operatorname{erfc}\left(x / 2 \sqrt{D_{L} t}\right)}{\operatorname{erfc}\left(\lambda \sqrt{\alpha_{S} / D_{L}}\right)}\right\} \frac{x}{X(t)} \exp \left\{\frac{x^{2}-X(t)^{2}}{4 t D_{L}}\right\} \rightarrow 1 .
$$

But for $x>X^{*}(t)+\delta$, we know that

$$
\exp \left\{\frac{x^{2}-X(t)^{2}}{4 t D_{L}}\right\} \rightarrow \infty \quad \text { as } D_{L} \rightarrow 0
$$

whence

$$
\frac{\operatorname{erfc}\left(x / 2 \sqrt{D_{L} t}\right)}{\operatorname{erfc}\left(\lambda \sqrt{\alpha_{S} / D_{L}}\right)} \rightarrow 0,
$$

and so, by (2c), $C(x, t) \rightarrow C_{\text {init }}$ uniformly for $x>X^{*}(t)+\delta$. Meanwhile,

$$
C_{x}(x, t)=-\frac{\left(C_{L}-C_{\text {init }}\right) e^{-x^{2} / 4 D_{L^{t}}}}{\sqrt{D_{L} t \pi} \operatorname{erfc}\left(\lambda \sqrt{\alpha_{S} / D_{L}}\right)}
$$

whence at $x=X(t)$,

$$
\begin{aligned}
C_{x}(X(t), t) & =-\frac{\left(C_{L}-C_{\text {init }}\right) e^{-\lambda^{2} \alpha_{S} / D_{L}}}{\sqrt{D_{L} t \pi} \operatorname{erfc}\left(\lambda \sqrt{\alpha_{S} / D_{L}}\right)} \\
& =\frac{-\left(C_{L}-C_{\text {init }}\right) \lambda \sqrt{\alpha_{S}}}{D_{L} \sqrt{t \pi} e^{\lambda^{2} \alpha_{S} / D_{L}} \operatorname{erfc}\left(\lambda \sqrt{\alpha_{S} / D_{L}}\right) \lambda \sqrt{\alpha_{S} / D_{L}}} \\
& \rightarrow \pm \infty
\end{aligned}
$$

as $D_{L} \rightarrow 0$, accordingly as $C_{L}<C_{\text {init }}$ or $C_{L}>C_{\text {init }}$. We have shown the following.

Proposition. As $D_{L} \rightarrow 0$, the explicit solution (2)-(4) has a limit. The concentration tends to $C_{\text {init }}$ everywhere except at the front, while the temperature and phase front tend to those of the classical two-phase Stefan problem with $T_{c r}=f_{S}\left(C_{\text {init }}\right)$. The phase diagram is no longer relevant.

A consequence of this convergence result is that the explicit solution may lose its physical meaning for very small $D_{L}$, as is shown in the following.

Corollary. For sufficiently small $D_{L}$ and points $x$ close to the front $X(t)$ on the liquid side $(x>X(t))$, the state $(C, T)$ of the material at $(x, t), t>0$, is between the liquids and solidus curves.

Indeed, as $D_{L} \rightarrow 0, T_{c r} \rightarrow f_{S}\left(C_{\text {init }}\right)$, while the interfaces converge to some $X(t)$. For $x>X(t)$ sufficiently close to $X(t), T(x, t) \geqslant T_{c r}$ and $C(x, t)<C_{\text {init }}$ when $D_{L}$ is sufficiently small. Then the monotonicity of $f_{S}(C)$ and $f_{L}(C)$ implies 


$$
f_{S}(C)<f_{S}\left(C_{\text {init }}\right)=T_{c r} \leqslant T<f_{L}(C),
$$

which says that $(x, t)$ lies neither in the liquid nor in the solid.

\section{Nomenclature}

$x$

$t$

$X$

$T$

$q$

$T_{c r}$

$C$

$K$

$c$

$\rho$

$\alpha=K /(c \rho)$

$H$

$D$

$T_{c r}^{A}$

$T_{c r}^{B}$

$f_{L}$

$f_{S}$

Subscripts

$L$

$S$ position $(m)$

time $(s)$

interface $(m)$

temperature $\left({ }^{\circ} \mathrm{C}\right)$

heat flux $\left(K J / M^{2}-S\right)$

melting temperature $\left({ }^{\circ} \mathrm{C}\right)$

concentration

thermal conductivity $\left(K J / m-s-{ }^{\circ} \mathrm{C}\right)$

specific heat $\left(K J / K g-{ }^{\circ} C\right)$

density $\left(K G / m^{3}\right)$

thermal diffusivity $\left(\mathrm{m}^{2} / S\right)$

latent heat $(K J / K g)$

material diffusion coefficient $\left(\mathrm{m}^{2} / \mathrm{s}\right)$

melt temperature of pure $A\left({ }^{\circ} \mathrm{C}\right)$

melt temperature of pure $B\left({ }^{\circ} \mathrm{C}\right)$

liquidus curve

solidus curve

liquid

solid

\section{REFERENCES}

[1] H. Carslaw and J. Jaeger, Conduction of heat in solids, 2nd edition, Oxford University Press, 1959

[2] A. B. Crowley and J. Ockendon, On the numerical solution of an alloy solidification problem, International Journal of Heat and Mass Transfer, 22, 941-947 (1979)

[3] L. Rubinstein, The stefan problem, American Mathematical Society Translations, 1967

[4] L. Rubinstein, Solidification of a binary alloy, Free Boundary Problems, Vol. 1, Proceedings of a seminar held in Pavia, Sept.-Oct., 1979, Instituto Nazionale di Alta Matematica F. Severi, Rome, 399-416, 1980

[5] D. Tarzia, An inequality for the coefficient $\sigma$ of the free boundary $s(t)=2 \sigma \sqrt{t}$ of the Neumann solution for the two-phase Stefan problem, Quarterly of Applied Math, 39, 491-497 (1982)

[6] D. G. Wilson, Existence and uniqueness for similarity solutions of one dimensional multi-phase Stefan problems, SIAM Journal of Applied Mathematics 5, 135-147 (1979) 